\title{
Aroma encapsulation and aroma delivery by oil body suspensions derived from sunflower seeds (Helianthus annus)
}

\author{
Ian D. Fisk • Rob S. T. Linforth • \\ Andrew J. Taylor · David A. Gray
}

Received: 1 November 2010/ Accepted: 19 February 2011/Published online: 18 March 2011

(C) The Author(s) 2011. This article is published with open access at Springerlink.com

\begin{abstract}
Oil bodies are small discrete cell organelles that can be found within oilseeds. Oil bodies have been investigated previously as a potential technology platform for use within the food industry, offering stable, antioxidant-enriched lipid-delivery systems. In this study, the use of oil bodies as a flavour delivery agent is evaluated. Fresh aromatized oil bodies show comparable headspace flavour intensity to phospholipid-stabilized emulsions when in a static equilibrium state, and when evaluated by dynamic headspace dilution, aromatized oil bodies showed a significantly stronger potential to maintain their headspace volatile concentration, which may indicate that oil bodies would offer greater retronasal flavour delivery than current commercial systems.
\end{abstract}

Keywords Emulsion - Encapsulation · Flavour · Lipid · Oil body $\cdot$ Sunflower

\section{Introduction}

Oil bodies are small $(0.2-2 \mu \mathrm{m}$ diameter $)$ discrete cell organelles that are commonly found as storage structures for neutral lipid within oilseeds for energy release during germination [1]. They consist of a core of triglyceride surrounded by a protective layer of phospholipid, in the form of a monolayer, and a coat of stabilizing proteins (e.g. oleosin, caleosin). The stabilizing proteins offer significant levels of structural stability to the oil bodies both in vivo

I. D. Fisk $(\varangle)$ - R. S. T. Linforth · A. J. Taylor · D. A. Gray Division of Food Science, University of Nottingham,

Sutton Bonington Campus, Sutton Bonington,

Leicestershire LE12 5RD, UK

e-mail: Ian.fisk@nottingham.ac.uk and ex vivo. Oil bodies' intrinsic chemical [2] and physical stability $[3,4]$ and the low cost of extraction, combined with the presence of natural antioxidants [3,5], illustrate the significant potential of oil bodies as stable food ingredients.

Oil bodies have been demonstrated as a potential technology platform for a number of key commercial offerings. Within the pharmaceutical industry, oleosin protein has been used as an effective carrier agent for recombinant proteins fused to its hydrophilic regions, allowing a simple route to purification and isolation. The use of oil bodies in food has been investigated by a number of authors who have characterized their physical-chemical properties and demonstrated the use of multilayer coatings on the surface and enzymatic surface modification as potential routes to further enhance structural stability [2-4, 6-11].

Prior to the addition of oil bodies into food systems, it is important to know how their presence will impact the release, retention and perception of volatile flavour compounds. In this investigation, such a study is presented detailing how oil bodies affect the release of flavour compounds and the potential application of oil bodies as flavour carrier agents.

The volatilization of flavour compounds from single phase matrices is dependent on many interacting factors. The main controlling parameters are the rate of mass transfer from the matrix [12], the physico-chemical parameters of the flavour molecule itself and various thermodynamic parameters (vapour pressure, solubility and activity coefficients). The partitioning of a flavour volatile between a matrix and its headspace will dictate the sensory perception of the volatile, and this is defined by the partition coefficient. The equilibrium concentration in both gaseous and liquid phases will be heavily dependent upon the chemical composition of the liquid phase, for example, 
the addition of lipids and proteins will change the relative solubility of the flavour compound and consequently the partition coefficient, leading to significant changes in headspace volatile availability and flavour perception.

When flavour volatiles are added to a cloud emulsion (oil-in-water suspension), a fraction of the flavour volatile will partition into the lipid phase, the level of which is dependent on the hydrophobicity of the flavouring. Very hydrophilic compounds will not partition into the lipid and will effectively be present at a higher concentration in the aqueous phase. A volatile of high hydrophobicity will partition significantly into the lipid phase and lead to a lower concentration in the aqueous phase; the air-emulsion partition coefficient will therefore reduce, and the volatile concentration in the air phase will reduce. The strength of this association was detailed by a range of authors [13-15] who suggest that the physical parameters of the flavour volatile and the oil fraction are the most significant factors when predicting the impact of lipid on a suspension of volatile in a cloud emulsion.

We propose that oil bodies could be a novel and effective method for encapsulating hydrophobic flavour compounds. In order to assess a flavour encapsulant, several parameters should be considered. These include the ability to retain flavour during storage and the ability to release flavour on demand. Despite all the work that has been carried out previously on oil body structure, oil body synthesis and oleosin biochemistry, no studies have been reported on their ability to deliver flavour within foods. There is also a lack of published literature detailing the commercial exploitation of oil bodies beyond pharmaceutical applications.

\section{Materials and methods}

Sunflower seed oil bodies were prepared by aqueous extraction, and the resulting oil body preparation was then washed with urea. This urea washed oil body (UWOB) preparation was used to understand the fundamental properties of purified oil body preparations in comparison with processed emulsions (at equivalent lipid content and droplet size distribution) when they interact with flavour compounds. All chemicals were sourced from Fisher Scientific, Loughborough, and were of analytical grade (>99.9\%) unless specified.

\section{Oil body isolation}

Oil bodies from dehulled sunflower seed were extracted and purified by the method of Tzen [16] with slight modifications. Seeds $(100 \mathrm{~g})$ in grinding medium $(0.5 \mathrm{~L}$, $10 \mathrm{mM}$ sodium phosphate buffer $\mathrm{pH} 7.5,0.6 \mathrm{M}$ sucrose) were ground in a laboratory blender (Kenwood BL315 full power for $60 \mathrm{~s}$ ). The resultant slurry was filtered through three layers of cheesecloth and the filtrate centrifuged in 400-mL batches (8000 RCF, $30 \mathrm{~min}, 5^{\circ} \mathrm{C}$ ). The upper layer was isolated using a chilled metal spatula and dispersed $(5 \mathrm{~mL})$ in washing buffer $(25 \mathrm{~mL}, 10 \mathrm{mM}$ sodium phosphate buffer, $\mathrm{pH} 7.5,5^{\circ} \mathrm{C}$ ) and then centrifuged at $5{ }^{\circ} \mathrm{C}$ (a swinging bucket rotor at $2000 \mathrm{RCF}$ for $20 \mathrm{~min}$ was used for all other centrifugations unless stated otherwise). The upper layer was isolated and designated as a waterwashed oil body preparation. This isolate was then further purified by suspending the water-washed preparation $(5 \mathrm{~mL})$ in urea-washing buffer $(25 \mathrm{~mL}, 9 \mathrm{M}$ urea, $10 \mathrm{mM}$ sodium phosphate buffer, $\mathrm{pH} 7.5$ ) and centrifuged at room temperature. The fat pad was isolated, re-suspended in washing buffer $(25 \mathrm{~mL}, 10 \mathrm{mM}$ sodium phosphate buffer, $\mathrm{pH} 7.5$ ) and centrifuged at room temperature to remove residual urea and extraneous proteins. After centrifugation, the centrifuge tube was chilled on ice for $5 \mathrm{~min}$ and the upper solidified phase (urea-washed oil body preparation) was removed, mixed with sodium azide (final concentration of sodium azide $0.02 \mathrm{mM}$ ) and stored at $5{ }^{\circ} \mathrm{C}$ under nitrogen. Replicates are three true replicates from different batches of seed from the same supplier purchased on the same day.

\section{Processed emulsion formulation}

An emulsion was formulated by mixing water $(0.78 \mathrm{~L})$ and soy lecithin (Sigma-Aldrich) $(0.03 \mathrm{~L})$ in a high shear mixer until dispersed, and sunflower oil (0.19 L) was then added dropwise under shear until an emulsion was formed. The crude emulsion was passed through a homogenizer (Emulsiflex C5, Glen Creston, Stanmore, UK) twice to form an emulsion that was uniform, mono-disperse, and had a droplet diameter of $1 \mu \mathrm{m}$. The processed emulsion was stored at $5{ }^{\circ} \mathrm{C}$ under nitrogen, and the replicates are three true replicates produced from the mixing of three separate preparations of water, lecithin and oil.

\section{Size distribution}

A Malvern Mastersizer S (Malvern Instrument, Malvern., England) equipped with a small sample dispersion unit and a 300RF lens was used to study the droplet diameter of oil body samples. The volume particle size distribution was estimated using the Malvern Mastersizer $\mathrm{S}$ polydisperse analysis model and 3NAD presentation code (real refractive index and imaginary refractive index were 1.095 and 0 , respectively, and dispersant refractive index was set at 1.33), and obscuration was maintained at $15 \%$ for all samples by dilution with $10 \mathrm{mM}$ sodium phosphate buffer, pH 7.5. 
Samples were introduced into the small sample dispersion unit after sonication ( $1 \mathrm{~min}$ ), and samples were allowed to equilibrate $(1 \mathrm{~min})$ prior to measurement to ensure samples had fully dispersed.

\section{Vacuum oven drying}

Samples were prepared in pre-dried containers and dried in a Gallenhamp vacuum oven at $-90 \mathrm{kPa}$ and $40{ }^{\circ} \mathrm{C}$ (48 $\mathrm{h}$ or until constant weight).

\section{Soxhlet lipid extraction}

Total fat was extracted by weighing oil body $(2 \mathrm{~g})$ or seed $(8 \mathrm{~g})$ samples into cellulose thimbles $(22 \mathrm{~mm} \times 80 \mathrm{~mm}$, Whatman, Fisher Scientific Ltd, Loughborough). Samples were capped with cotton wool and placed into Soxhlet glassware. Two hundred millilitre of $\left(40-60{ }^{\circ} \mathrm{C}\right)$ petroleum ether was used to extract total fat by refluxing on a heating plate for $18 \mathrm{~h}$.

\section{Static headspace analysis by APcI-MS}

Static headspace volatile analysis was performed according to Linforth [17]. In brief, a direct injection mass spectrometer (APcI-MS, MS-Nose ${ }^{\mathrm{TM}}$, Micromass-LCZ, Micromass, Altrincham, UK) was used to evaluate the headspace gas concentration of a range of volatile compounds, and samples $(10 \mathrm{~mL})$ were placed in a capped Schott Bottle (volume $=123 \mathrm{~mL}$ ) with two plugged holes in the lid (sampling port, gas dilution port). After equilibrium $\left(2 \mathrm{~h}, 25^{\circ} \mathrm{C}\right)$, the sampling plug was removed and the interface probe for the APcI-MS was passed through the hole. The interface sampled (sampling gas flow rate $10 \mathrm{~mL} \mathrm{m^{-1 }}$ ) the headspace $(30 \mathrm{~s}$ ) and measured the concentration of volatiles present in the headspace. Calibration was achieved by direct injection of the volatile in hexane into the mass spectrometer, and temperature was maintained throughout at $25 \pm 2{ }^{\circ} \mathrm{C}$.
(a) $K_{a e}=100 \times \frac{C_{a}}{C_{e}}$
(b) $K_{a w}=100 \times \frac{C_{a}}{C_{w}}$
(c) $\mathrm{RHI} \%=100 \times \frac{C_{E}}{C_{W}}$
(d) $n \mathrm{HI}_{t=i}=100 \times \frac{C_{E(t=i)}}{C_{E(t=0)}}$

Equation 1 (a) Air-emulsion and (b) air-water partition coefficient $(K)$, (c) relative headspace intensity (RHI\%) and (d) normalized headspace intensity $(\mathrm{nHI})$, where $C_{x}$ is the concentration in $a=$ air, $e=$ emulsion, $w=$ water,
$E=$ headspace above emulsion, $W=$ headspace above water, at time $t=i$.

The variation in relative headspace intensity was measured separately for a range of volatiles (carvone (98\%), 2-nonanone (99\%), methyl hexanoate (99\%), 2,5 dimethyl pyrazines $(>98 \%)$, ethyl butyrate $(98 \%)$, menthol $(99 \%)$, ethyl octanoate (99\%) and limonene $(96 \%)$ ) which were chosen to cover a range of hydrophobicities, physicochemical properties and volatilities in both an oil body suspension and a processed emulsion. Headspace concentration was directly compared and relative headspace intensity (\% RHI) calculated, as per Doyen [14] (Eq. 1).

Solutions of volatile (10 ppm w/v) were prepared separately by shaking (15 min, SF1 flask shaker, Stuart Scientific, Redhill, UK). Oil body suspensions and emulsions were then diluted with volatile solution and additional water to achieve a final lipid concentration of $1 \% \mathrm{w} / \mathrm{w}$ and a volatile concentration of $1 \mathrm{ppm}$ (100 ppm for dimethyl pyrazine). Linearity of the MS response was tested by preparation of a standard curve of a range of volatile concentrations in buffer for each volatile. Data were analysed by analysis of variance, $p<0.05$.

All volatiles were sourced from Sigma-Aldrich Company Limited, Dorset, England, apart from Limonene which was sourced from Acros Organics, now Thermo Fisher Scientific, New Jersey, US.

\section{Dynamic headspace analysis by APcI-MS}

Dynamic headspace analysis was performed according to the method of Doyen [14]. Samples (100 mL) were dispersed in solution and stored in a Schott bottle $(123 \mathrm{~mL})$, allowed to equilibrate $(4 \mathrm{~h})$ and then the headspace measured for volatile concentration during dynamic headspace dilution. Dynamic headspace dilution was achieved by passing nitrogen gas $\left(70 \mathrm{~mL} \mathrm{m^{-1 }}\right)$ through a dilution gas port, and at the same time, the gas exiting from the sampling gas port was measured by APcI-MS (Sample flow $70 \mathrm{~mL} \mathrm{m^{-1 }}$, cone voltage $20 \mathrm{~V}$ ). Headspace dilution was continued for $10 \mathrm{~min}$, and temperature was maintained throughout at $25 \pm 2{ }^{\circ} \mathrm{C}$. Data were analysed by analysis of variance and Tukey's HSD, $p<0.05$.

\section{Results and discussion}

UWOB suspensions and processed emulsions were stable during volatile analysis and did not destabilize. Average (volume) particle size distribution for UWOB was $1.5 \pm 0.04 \mu \mathrm{m}$. 
Evaluation of headspace volatile concentration in an equilibrium state

The ability of UWOB to modify the headspace concentration of a range of flavour volatiles was assessed. This was achieved by combining a suspension of oil bodies and flavour compounds in an aqueous buffer and then by measuring the concentration of flavour volatile in the headspace at equilibrium (Table 1).

The static headspace results (Table 1) for all 8 flavour compounds show a close correlation between the relative headspace intensity above the oil body suspension and the processed emulsion. This is consistent with the work reported by Carey [15] who concluded that the major determining factors effecting \% RHI in a cloud emulsion were the oil fraction and the molecular characteristics of the volatile. The lipid content was fixed at $10 \%$ in all samples. Analysis of variance showed that there were no statistically significant differences $(p=0.4)$ between the emulsion type (UWOB and the processed emulsion) but a significant impact of the volatile $(p<0.001)$ was identified. In addition, headspace \% RHI data were compared with predicted \% RHI values produced from the Buttery model [13]. The model \% RHI value correlated well with the experimental data (correlation coefficient $=0.86$ ).

UWOB suspensions are comparable to processed emulsions at partitioning volatiles in a static headspace situation. This suggests that at ambient temperatures, any residual proteins associated with oil bodies do not affect the partitioning of flavour compounds whilst in an equilibrium state (Fig. 1).

Evaluation of headspace volatile concentration during dynamic headspace dilution

Although the static headspace volatile concentrations above UWOB suspensions are comparable to phospholipid-stabilized emulsions, the simple partitioning of

Table 1 Static headspace equilibrium \% RHI for 8 flavour volatiles in processed emulsions (PE) and UWOB suspensions (OB) (lipid content $=10 \%, n=3$, average \pm standard deviation)

\begin{tabular}{lllll}
\hline \% RHI & Carvone & 2-nonanone & $\begin{array}{l}\text { Methyl } \\
\text { hexanoate }\end{array}$ & $\begin{array}{l}\text { 2,5 Dimethyl } \\
\text { pyrazine }\end{array}$ \\
\hline OB & $42 \pm 3.7$ & $10 \pm 0.44$ & $43 \pm 2.8$ & $97 \pm 10$ \\
PE & $41 \pm 8.5$ & $11 \pm 0.49$ & $52 \pm 3.1$ & $94 \pm 12$ \\
$\log$ P & 3.1 & 1.9 & 2.3 & 4.8 \\
\hline$\%$ RHI & Ethyl butyrate & Menthol & Ethyl octanoate & $(+)$-Limonene \\
\hline OB & $61 \pm 6.9$ & $12 \pm 1$ & $1.6 \pm 0.19$ & $17 \pm 0.35$ \\
PE & $72 \pm 2.7$ & $14 \pm 1.9$ & $1.7 \pm 0.17$ & $8.9 \pm 1.9$ \\
$\log$ P & 1.9 & 2.3 & 3.1 & 4.8 \\
\hline
\end{tabular}

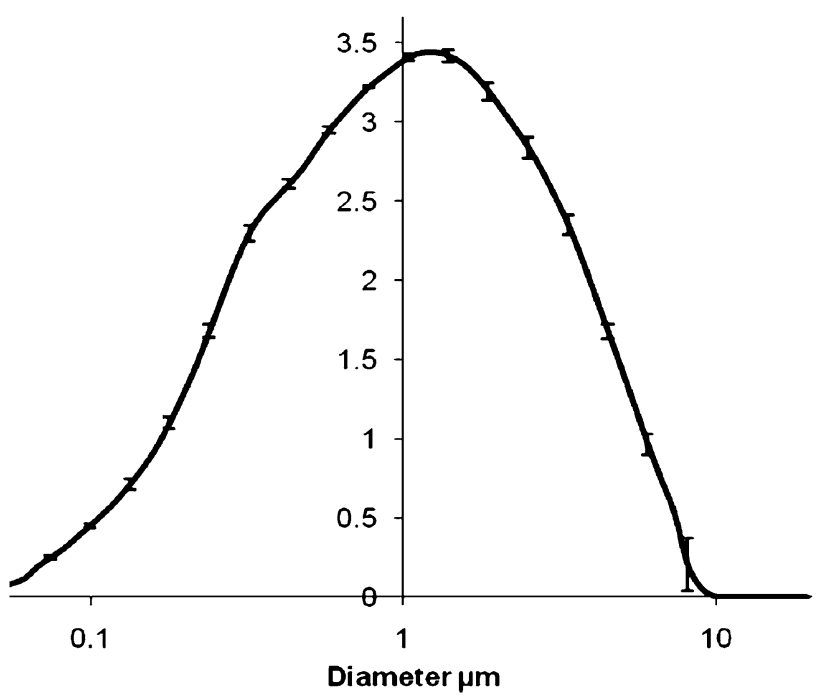

Fig. 1 UWOB droplet size distribution (\% volume frequency $\pm \mathrm{SD}$ ) prior to analysis. There was no significant change in particle size distribution during volatile analysis $(n=3)$

flavour volatiles between a solution and its headspace at equilibrium does not encompass all the facets of flavour release.

To explore the more complex dynamic association of flavour volatiles to the emulsion and the associated proteins and phospholipids, the rate at which an oil body suspension and a processed emulsion can maintain their headspace volatile concentration with constant headspace dilution was investigated. This was completed with limonene $(0.015$ and $1 \%$ lipid content) as the flavour volatile of interest; limonene was chosen as it is a common flavour ingredient, is strongly lipophilic $(\log \mathrm{P}=3.6)$ and has commercial relevance to cloud emulsions.

On dilution of the equilibrium headspace with a clean airflow, the normalized headspace intensity of flavouring above the buffer sample rapidly reduced to below $50 \%$ of its initial concentration (Fig. 2). The oil body suspension maintained the headspace concentration consistently at a higher average normalized headspace intensity (Table 2) over the $10 \mathrm{~min}$ of sampling and took longer to reach equilibrium (Fig. 2). The processed emulsion sample had an intermediate normalized headspace intensity profile. The difference is more significant $(p<0.05)$ at $1 \%$ lipid content, and although no consistent statistically significant difference can be measured between the UWOB and the PE at $0.0015 \%$ lipid, a clear trend can be observed Table 2 .

Doyen's [14] study on the release of ethyl octanoate from lipid emulsions (lipid concentrations 0-19 g lipid $\mathrm{L}^{-1}$ ) concluded that the normalized headspace intensity above lipid emulsions had a greater resistance to dilution than in a comparable system containing only water and volatile. Doyen also suggested that, in addition to maintaining the volatiles headspace concentration at a higher 


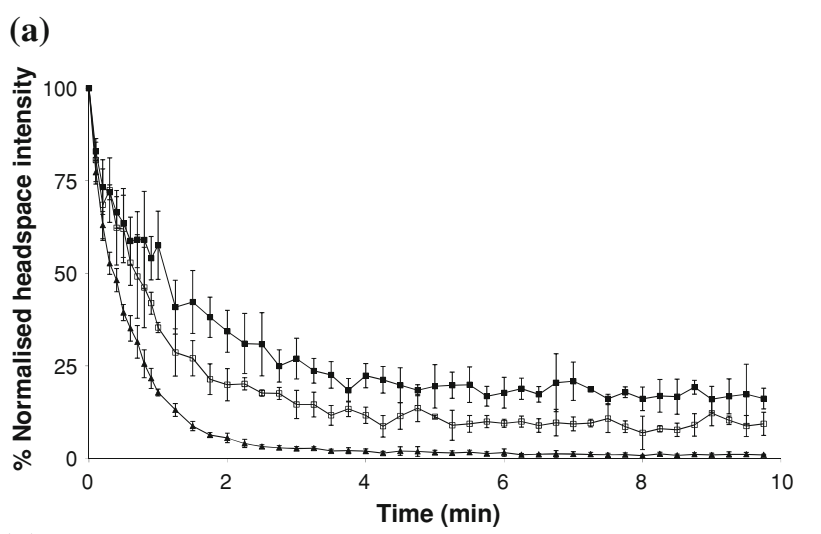

(b)

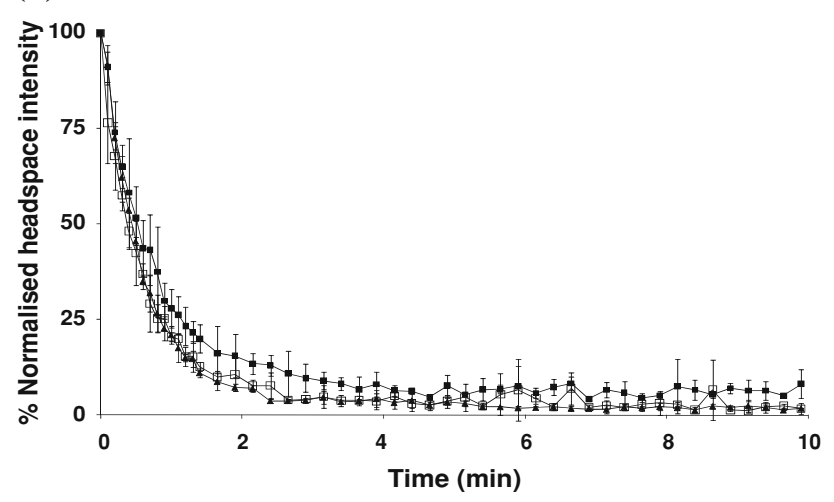

Fig. 2 Normalized time-course profiles for dynamic headspace dilution measurements of limonene in an aqueous suspension of UWOB (filled square), processed emulsion (unfilled square) and buffer (filled triangle) for a limonene in a $1 \%$ lipid sample and b limonene in a $0.015 \%$ lipid sample

normalized headspace intensity, the time to reach equilibrium was shorter in a lipid emulsion than above a purely aqueous system; this is consistent with data presented in Fig. 2.

In an aqueous system, the rate of headspace volatile replenishment is primarily dependent upon the rate of movement of flavour molecules across the air-water interface. If the headspace concentration is low compared to that of the solution (as is the case for a volatile of $K_{a w}=10^{-5}$ ) (Eq. 1), the headspace can be rapidly replenished as only a limited number of flavour molecules are required to volatilize and transfer into the headspace to restore equilibrium. In a system of higher headspace concentration relative to the solution concentration (as is the case for a volatile of $K_{a w}=10^{-2}$ ), a larger number of flavour molecules will be required to transfer into the headspace from the solution to restore equilibrium, thus reducing the rate at which the volatile concentration in the headspace can be replenished with dilution, resulting in a lower normalized headspace intensity.

When lipid is suspended in an aqueous solution producing an emulsion, the solubility of any lipophilic flavour volatile increases. This increase in solubility reduces the original $K_{a w}$ to a lower $K_{a e}$ (Eq. 1). This reduction in the partition coefficient results in a reduced headspace concentration and a volatile headspace concentration that can be replenished more rapidly giving flavour volatiles in the emulsion system a higher normalized headspace intensity.

This increase in normalized headspace intensity was observed for limonene in both the processed emulsion and oil body suspensions. The reasons why volatiles in an oil body suspension have a greater normalized headspace intensity than those in an processed emulsion are unknown. For an oil-in-water emulsion to have a greater normalized headspace intensity, it is generally accepted that the rate of mass transfer across the air-emulsion interface must be greater.

One possible explanation for the proposed increased mass transfer rate would be that oil bodies and their associated proteins are present at a higher concentration at the surface than the bulk liquid reservoir, forming an invisible boundary layer. This boundary layer would act as a reservoir for flavour volatiles and reduce the effective air-emulsion partition coefficient between this boundary layer and the headspace (during headspace dilution). The layer therefore allows a more rapid transfer of flavour molecules into the headspace and subsequently maintains the headspace concentration of the flavour volatile at a higher normalized headspace intensity during dilution [18]. In a static system, the boundary layer may still form but the

Table $2 \%$ Normalized headspace intensity of products at time points during dynamic headspace dilution

\begin{tabular}{llllllccccc}
\hline & $1 \mathrm{~min}$ & $2 \mathrm{~min}$ & $3 \mathrm{~min}$ & $4 \mathrm{~min}$ & $5 \min$ & 6 min & 7 min & 8 min & $9 \mathrm{~min}$ & $10 \mathrm{~min}$ \\
\hline OB(HIGH) & $57.56^{A}$ & $34.33^{A}$ & $26.96^{A}$ & $22.36^{A}$ & $19.49^{A}$ & $17.74^{A}$ & $20.86^{A}$ & $16.10^{A}$ & $15.98^{A}$ & $16.16^{A}$ \\
PE(HIGH) & $35.35^{B}$ & $19.93^{B}$ & $14.58^{B}$ & $11.65^{B}$ & $11.28^{B}$ & $9.50^{A B}$ & $9.31^{B}$ & $6.92^{B}$ & $12.22^{A B}$ & $9.35^{B}$ \\
OB(LOW) & $27.94^{B C}$ & $15.53^{B C}$ & $9.73^{B C}$ & $8.10^{B C}$ & $7.72^{B C}$ & $7.60^{A B}$ & $4.26^{B C}$ & $5.13^{B}$ & $7.03^{B C}$ & $8.14^{B C}$ \\
PE(LOW) & $20.49^{C}$ & $10.64^{B C}$ & $4.19^{C}$ & $3.60^{C}$ & $3.65^{B C}$ & $6.59^{A B}$ & $2.09^{C}$ & $3.16^{B}$ & $1.28^{D}$ & $1.71^{C D}$ \\
Buffer(1) & $21.00^{C}$ & $7.25^{C}$ & $3.89^{C}$ & $4.26^{C}$ & $3.51^{C}$ & $1.87^{B}$ & $1.65^{C}$ & $2.24^{B}$ & $2.02^{C D}$ & $1.78^{C D}$ \\
Buffer(2) & $17.79^{C}$ & $5.55^{C}$ & $2.67^{C}$ & $1.97^{C}$ & $1.63^{C}$ & $1.58^{B}$ & $1.19^{C}$ & $0.77^{B}$ & $0.96^{D}$ & $0.98^{D}$ \\
\hline
\end{tabular}

$\overline{A B C}$ samples with different letters within a column are significantly different, ANOVA-Tukey's HSD, $p<0.05, n=3 ; O B$ UWOB, $P E$ processed emulsion, Buffer water only, Low lipid content $0.015 \%$, High lipid content $1 \%$; for buffer (1) and (2) indicates replicate experiments 
effect of the apparent increased interfacial lipid content may not be observed since the static situation reflects bulk phase properties. This would require the oil body-associated proteins to be surface active and be more thermodynamically stable at the air-emulsion interface than in the bulk solution; this was recently proved by Roux [19], who showed that the interaction of oleosin with phospholipid stabilized the air-water interface.

The implication of this finding is that oil body suspensions would have a greater headspace concentration of volatile compounds compared with a comparable phospholipid-stabilized emulsion when a flow of air is passing over the surface. This characteristic 'controlled release' from oil body suspensions could improve flavour retention in a food product and retronasal flavour perception on consumption. If oil bodies are surface active, this would contribute to a further increase in retronasal flavour delivery, and as oil bodies would partially adsorb to the buccal epithelia and contribute an elevated flavour volatile concentration retronasally, a similar phenomenon has previously been shown by Linforth and co-workers [20].

Cook [21] looked at the effect of physical properties on retronasal flavour release and showed that the viscosity of a consumed solution did not affect retronasal flavour delivery. Cook hypothesized, within the study, that it was the interfacial properties of the solution that are most significant in controlling flavour concentration retronasally. This was also documented by Linforth and co-workers [22] who also showed that a reduction in partition coefficient increases efficiency of volatile delivery, resulting in a volatile concentration in-nose greater than expected on a basis of $K_{a e}$.

\section{Conclusion}

From the results described above, oil bodies act as effective flavour carrier agents. Oil bodies offer enhanced flavour delivery through elevated headspace flavour persistence; this offers significant potential for future studies on the sensory perception of aromatized oil body systems.
Open Access This article is distributed under the terms of the Creative Commons Attribution Noncommercial License which permits any noncommercial use, distribution, and reproduction in any medium, provided the original author(s) and source are credited.

\section{References}

1. Tzen JTC, Cao YZ, Laurent P, Ratnayake C, Huang AHC (1993) Plant Physiol 101:267-276

2. Fisk ID, White DA, Lad M, Gray DA (2008) Eur J Lipid Sci Tech 110:962-968

3. White DA, Fisk ID, Gray DA (2006) J Cereal Sci 43:244-249

4. Iwanaga D, Gray DA, Fisk ID, Decker EA, Weiss J, McClements DJ (2007) J Agr Food Chem 55:8711-8716

5. Fisk ID, White DA, Carvalho A, Gray DA (2006) J Am Oil Chem Soc 83:341-344

6. White DA, Fisk ID, Mitchell JR, Wolf B, Hill SE, Gray DA (2008) Food Hydrocolloid 22:1224-1232

7. White DA, Fisk ID, Makkhun S, Gray DA (2009) J Agr Food Chem 57:5720-5726

8. Nikiforidis CV, Kiosseoglou V (2009) J Agr Food Chem 57:5591-5596

9. Nikiforidis CV, Kiosseoglou V (2010) J Agr Food Chem 58:527-532

10. Chen YM, Ono T (2010) J Agr Food Chem 58:7402-7407

11. Chen BC, McClements DJ, Gray DA, Decker EA (2010) J Agr Food Chem 58:9259-9265

12. Harrison M, Hills BP (1997) Int J Food Sci Tech 32:1-9

13. Buttery RG, Guadagni DG, Ling LC (1973) J Agr Food Chem 21:198-201

14. Doyen K, Carey M, Linforth RST, Marin M, Taylor AJ (2001) J Agr Food Chem 49:804-810

15. Carey ME, Asquith T, Linforth RST, Taylor AJ (2002) J Agr Food Chem 50:1985-1990

16. Tzen JTC, Peng CC, Cheng DJ, Chen ECF, Chiu JMH (1997) J Biochem 121:762-768

17. Linforth RST (1999) Apparatus and methods for the analysis of trace constituents of gasses. US Patent 5,869,344

18. Harrison M, Hills BP, Bakker J, Clothier T (1997) J Food Sci 62:653-664

19. Roux E, Baumberger S, Axelos MAV, Chardot T (2004) J Agr Food Chem 52:5245-5249

20. Linforth RST, Blissett A, Taylor AJ (2005) J Agr Food Chem 53:7217-7221

21. Cook DJ, Hollowood TA, Linforth RST, Taylor AJ (2003) Chem Senses 28:11-23

22. Linforth R, Martin F, Carey M, Davidson J, Taylor AJ (2002) J Agr Food Chem 50:1111-1117 